\title{
Structural Variations in Nanocrystalline Nickel Films
}

\author{
Pratibha L. Gai ${ }^{1}$, Rahul Mitra ${ }^{2 *}$, and Julia R. Weertman ${ }^{2}$ \\ ${ }^{1}$ DuPont Central Research,Wilmington DE 19880-0356 and also at, University of Delaware \\ ${ }^{2}$ Northwestern University, Department of Materials Science, Evanston, IL 60208 \\ * Current address: Defence Metallurgical Research Laboratory, Hyderabad, India.
}

Nanocrystalline metals are important because they exhibit exceptionally high strengths and therefore have generated considerable interest [1]. Nanocrystalline films have been synthesized by magnetron sputtering under various conditions. Some samples were grown on holey carbon filmed copper grids at liquid nitrogen temperatures using a substrate bias of $-150 \mathrm{~V}$ (designated as samples I). Application of bias enhances sputtering of the film by argon ions and leads to densification and equiaxed grain morphology. Another set of samples was grown on $\mathrm{NaCl}$ substrates (with an adjacent Si substrate for thickness calibration) without the application of substrate bias ( $0 \mathrm{~V}$ bias), also at liquid nitrogen temperatures (referred to as samples II).

We have obtained structural and chemical data at atomic resolution and on the nanoscale, respectively, using FEI FE(S)TEM Tecnai and CM30 Environmental-HRTEM (ETEM)[2] instruments. The data are quite informative. Previous studies [1] have shown that both type I and II samples have $<111>$ fiber texture with weaker $<111>$ texture in sample I. Our atomic structural studies of both the samples show that they contain nanocrystals with (111), as well as some with (100) and (110) surface planes. In sample I, HRTEM and dark field TEM show grain sizes between a few nanometers $(\mathrm{nm})$ to $\sim$ a few tens of $\mathrm{nm}$, and grains exhibit twins and interfacial defects [FIG.1]. The high internal strains indicate that these could form as the films grow from the original nuclei and the growing nuclei meet at boundaries. In the atomic images, nanoporosity with pores of $1 \mathrm{~nm}-2 \mathrm{~nm}$ is observed especially along grain boundaries. The lattice spacings and nano-probe analyses using X-ray spectroscopy (EDX) are consistent with $\mathrm{Ni}$, with no impurity segregation at the grain boundaries. We have not observed amorphous intergranular regions [FIG. 2]. There are interfacial dislocations and Moire fringes due to overlapping grains.

In sample II, nanoparticles have sizes in the range of a few $\mathrm{nm}$ to a few tens of $\mathrm{nm}$ and have increased porosity compared to sample I. Amorphous regions up to a few $\mathrm{nm}$ in extent are found in the interface regions between the nanocrystals [arrowed in FIG.3], confirmed by atomic resolution imaging and extensive sample tilting to different crystallographic orientations. Some Ni nanoparticles (clusters) are also observed in amorphous regions near the edge of the films. The FE-STEM nanoprobe analyses using the Tecnai have shown that intergranular regions are primarily amorphous Ni-rich regions and no impurities have thus far been detected within the detection limits of the instrument. This direct evidence indicates that the crystallization is not completed in sample II. In general, our studies have shown single Ni crystals and fewer intra and inter-granular defects compared to sample I. The origin and the development of the crystallization process with and without the substrate bias voltage have been inferred from the results.

References:

[1] R. Mitra, R.A. Hoffman, A. Madan and J.R. Weertman, J. Mater. Res. 16 (No.4) (2001)1010.

[2] P.L. Gai, Adv. Materials. 10 (1998) 1259. 


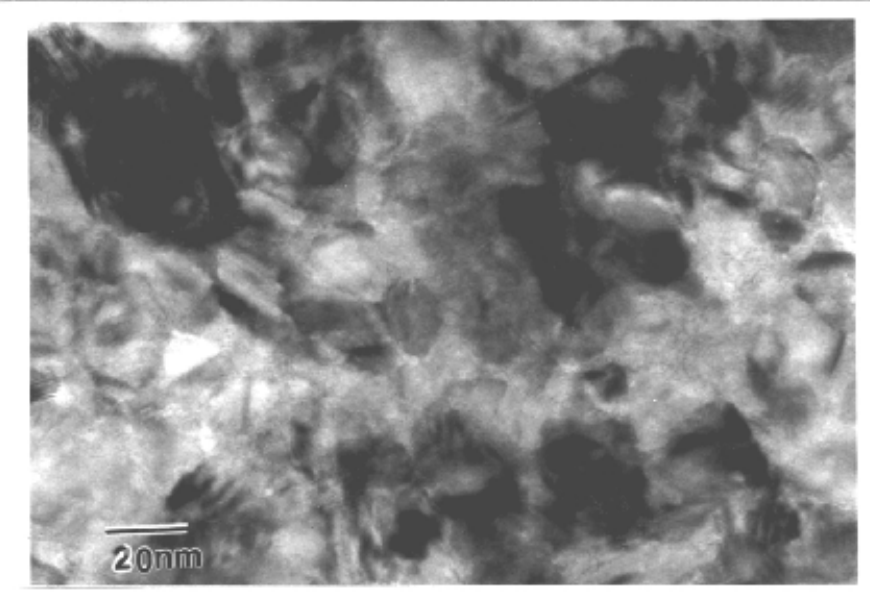

FIG. 1: varying grain sizes in nanocrystalline sample I.

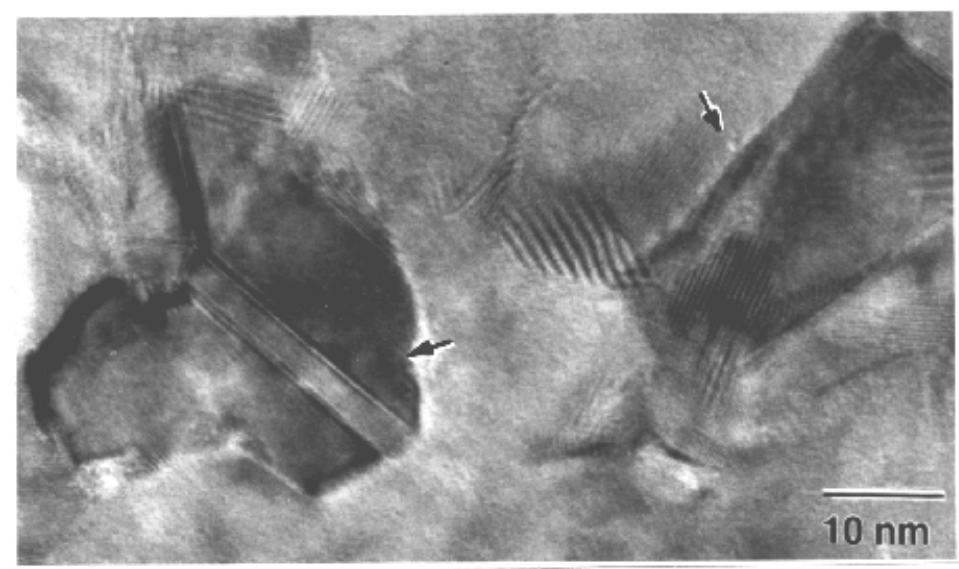

FIG.2: Nanocrystalline $\mathrm{Ni}$ with defects in sample I with a substrate bias of $-150 \mathrm{~V}$.

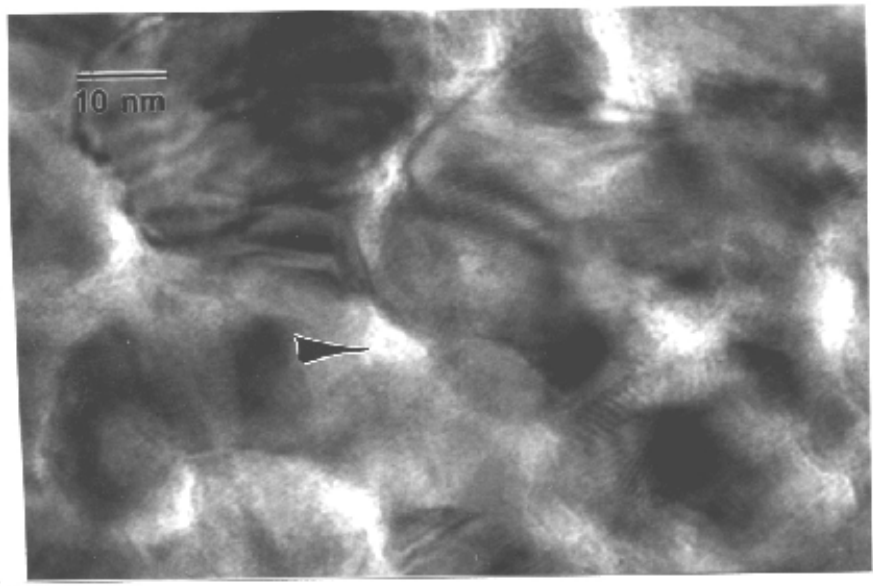

FIG.3: Nanoscale amorphous regions at grain boundaries in samples with no substrate bias 